\title{
Could C-Reactive Protein/Albumin Ratio be an Indicator of Activation in Axial Spondyloarthritis?
}

\author{
Melih Pamukcu ${ }^{1}$ and Tugba Izci Duran ${ }^{2}$ \\ ${ }^{1}$ Department of Clinic of Rheumatology, Dışkapl Education and Research Hospital, Health Sciences University, Turkey \\ ${ }^{2}$ Department of Internal Medicine, Division of Rheumatology, Medical Faculty, Ondokuz Mayis University, Turkey
}

\begin{abstract}
Objectives: To evaluate the relationship of C-reactive protein (CRP)/albumin ratio (CAR), neutrophil/lymphocyte ratio (NLR), platelet/lymphocyte ratio with Bath Ankylosing Spondylitis Disease Activity Index (BASDAl) in axial spondyloarthritis (AxSpA).

Study Design: Cross-sectional study.

Place and Duration of Study: Clinic of Rheumatology, Dışkapı Education and Research Hospital, Health Sciences University, Turkey during January 2020-2021.

Methodology: One-hundred and nine patients aged >18 years were included. Age, gender, duration of disease, BASDAI scores were recorded. CRP, erythrocyte sedimentation rate (ESR), albumin values, leukocyte, neutrophil, lymphocyte, platelet counts were determined.

Results: The mean age and median disease duration of the patients were $45.03 \pm 10.63$ and $5(2-6.5)$ years, respectively. Among these, 28 (58.3\%) female and 44 (72.1\%) male patients were found to be HLA-B27 positive. BASDAl $\geq 4$ was observed in 61 (56\%) patients and <4 in 48 (44\%) patients. CRP, albumin, ESR, NLR and CAR values between the patient groups were significantly different. There was high correlation between BASDAI and CAR. There was a moderate correlation between CAR and BASDAl in the patient group with normal acute phase reactants.

Conclusion: There is a significant correlation between CAR value and composite score such as BASDAl, which is commonly used to assess systemic inflammation in Ax-SpA. These methods are reliable, recognised by reimbursement institutions and include measurements that physicians can use practically in routine outpatient clinics.
\end{abstract}

Key Words: Spondylitis, Ankylosing, HLA-B27 antigen, C-reactive protein/metabolism, Serum albumin/metabolism, Neutrophils, Lymphocytes, Platelet count.

How to cite this article: Pamukcu M, Duran TI. Could C-Reactive Protein/Albumin Ratio be an Indicator of Activation in Axial Spondyloarthritis?. J Coll Physicians Surg Pak 2021; 31(05):537-541.

\section{INTRODUCTION}

Axial spondyloarthritis (Ax-SpA) is a chronic disease that causes inflammation in the spine, sacroiliac and large peripheral joints, specific entheses, and extra-articular structures such as the anterior uvea and aorta. ${ }^{1}$ Intervertebral and facet joint ankylosis occurs due to new bone formation in the axial skeleton, leading to functional insufficiency. It is magnetic resonance imaging (MRI) that enables early diagnosis. Early diagnosis and administration of appropriate drug therapy have higher rates of clinical remission. Therefore, there is an unmet need for the identification of biomarkers associated with Ax-SpA. ${ }^{1}$

Correspondence to: Dr. Tugba Izci Duran, Department of

Internal Medicine, Division of Rheumatology, Medical

Faculty, Ondokuz Mayıs University, Turkey

E-mail: drtugbaizciduran@gmail.com

Received: February 18, 2021; Revised: March 24, 2021;

Accepted: April 15, 2021

DOI: https://doi.org/10.29271/jcpsp.2021.05.537
Once the treatment is initiated, it is necessary to monitor its effect meticulously. However, this is currently limited largely to the patient's self-reported measurements for the severity of spinal symptoms and the levels of the most investigated and relatively insensitive serological marker $\mathrm{C}$-reactive protein (CRP). Serial assessment of MRI scans is not feasible for objective evaluation of inflammation. Elevation in CRP is independently associated with spinal radiographic progression in AxSpA. ${ }^{2}$

In Ax-SpA, it is important to evaluate the disease activity of the patient in order to direct the treatment and obtain information regarding the progression of the disease. ${ }^{3} \mathrm{CRP}$ and erythrocyte sedimentation rate (ESR) are frequently used markers to evaluate acute phase response due to their reliability and cost effectiveness. ${ }^{4}$ However, they are not considered sufficient. Although they are frequently used as acute phase reactants (APR) and reflect disease activity in Ax-SpA, their levels may be within normal limits in approximately one-third of the patients even if the disease is active. ${ }^{3} \mathrm{CRP}$ is a representative APR synthesised mainly by hepatocytes under the control of proinflamma- 
tory cytokines, particularly interleukin-6 (IL-6). ${ }^{5}$ Albumin is a negative APR and an acute inflammation marker. Low albumin levels are associated with the severity of inflammatory response. ${ }^{6}$

In the evaluation of patients with $\mathrm{Ax}-\mathrm{SpA}$, there is a need for simple and rapid scales that can be used in outpatient clinic conditions in daily practice in addition to composite score such as Bath Ankylosing Apondylitis Disease Activity Index (BASDAI) that has proven validity and reliability. ${ }^{7}$ In recent studies, it has been reported that platelet-lymphocyte ratio (PLR) and neutrophil-lymphocyte ratio (NLR) are important in demonstrating systemic inflammation. ${ }^{8,9}$ Similarly, as an inflammation marker, the CRP-Albumin ratio (CAR) has been shown to have prognostic significance in inflammatory processes such as cardiovascular disease and stroke. ${ }^{10,11}$ CAR value reflects inflammation more accurately compared to isolated serum CRP level. ${ }^{11,12}$ Although it is a valuable indicator for reflecting systemic inflammation, the relationship of BASDAI to CAR in AxSpA has not been previously investigated. Therefore, this study was done to investigate the relationship of CAR value with BASDAl composite score in patients with Ax-SpA.

\section{METHODOLOGY}

In this cross-sectional study, 109 patients $>18$ years of age who were regularly followed up in the rheumatology clinic during January 2020 - 21 and diagnosed with Ax-SpA based on the Assessment of SpondyloArthritis International Society (ASAS) criteria, ${ }^{13}$ were included. Pregnant and breastfeeding patients and those who had other diseases like malignancies, active hepatitis, chronic organ failure, and other chronic inflammatory diseases, were excluded. Written informed consent was obtained from each patient, and the study was conducted in accordance with the Helsinki Declaration principles.

Demographic data, duration of disease, and BASDAI score of the patients with Ax-SpA were recorded. ESR (0 - 20 mm/hour), CRP (normal range $0-5 \mathrm{mg} / \mathrm{dL}$ ), and albumin ( $\mathrm{g} / \mathrm{dL}$ ) values were determined based on laboratory analysis. Neutrophil $(\mathrm{K} / \mu \mathrm{L})$, lymphocyte $(\mathrm{K} / \mu \mathrm{L})$ and platelet $(\mathrm{K} / \mu \mathrm{L})$ counts were determined from based on complete blood count analysis. The CAR value were calculated by dividing the CRP level by the albumin level. And also PLR and NLR values were calculated by dividing platelet count by lymphocyte count, and neutrophil count by the lymphocyte count, respectively.

BASDAI was used to evaluate disease-specific symptoms such as fatigue, spinal and peripheral joint pain, swelling, and morning stiffness and interpreted based on a score ranging from 0 to 10. It has been shown that the Turkish version of BASDAl is sensitive to changes, valid and reliable. ${ }^{14}$ The data of the two groups, which were divided based on BASDAI score (BASDAI $\geq 4$ = high activity, $<4=$ low activity), were compared statistically.

SPSS (Statistical Package for Social Sciences) version 22.0 software was used for statistical analysis of the data. Descriptive statistical data were presented as frequency (percentage), mean \pm standard deviation (SD) and median (IQR: $25^{\text {th }}$ percentile- $75^{\text {th }}$ percentile). The distribution characteristics of numerical variables were evaluated using the Shapiro-Wilk test. Independent - Samples T-test was used for intergroup comparisons of numerical variables with normal distribution, while MannWhitney U-test was used for those without normal distribution. Chi-square test was used for evaluating categorical data and Spearman's correlation analysis test (rho) was used for correlation analysis. For the interpretation of correlation coefficients, low $(0.05-0.30)$, low-to-moderate $(0.30-0.40)$, moderate $(0.40-0.60)$, good $(0.60-0.70)$, very good $(0.70-0.75)$, and high $(0.75$ - 1.00) correlation ranges, which were recommended by Hayran, were used as reference. ${ }^{15} p<0.05$ was considered statistically significant.

\section{RESULTS}

The mean age of the 109 patients (48 females, 61 males) included in the study was $45.03 \pm 10.63$ years, and the median disease duration was $5(2-6.5)$ years. The numbers of female and male patients who were found to be HLA-B27 positive were $28(58.3 \%)$ and 44 (72.1\%), respectively. The main characteristics of the Ax-SpA patients included are shown in Tablel.

Table I: Characteristics of Ax-SpA patients.

\begin{tabular}{|c|c|c|}
\hline \multicolumn{2}{|l|}{ Parameter } & Patient Group $(n=109)$ \\
\hline \multicolumn{2}{|c|}{ Age, years (mean \pm SD) } & $45.03 \pm 10.63$ \\
\hline \multicolumn{2}{|c|}{ Female, $\mathrm{n}(\%)$} & $48(44 \%)$ \\
\hline \multicolumn{2}{|c|}{ HLA-B27 positive, n (\%) } & $72(66.1 \%)$ \\
\hline \multicolumn{2}{|c|}{ Disease duration, years } & $5(2-6.5)$ \\
\hline \multicolumn{2}{|c|}{ BASDAI 0-10, (mean \pm SD) } & $3.94 \pm 1.14$ \\
\hline \multicolumn{2}{|c|}{$\mathrm{CRP}, \mathrm{mg} / \mathrm{L}$} & $5.41(2.58-10.54)$ \\
\hline \multicolumn{2}{|l|}{$\mathrm{ESR}, \mathrm{mm} / \mathrm{h}$} & $9(4-16)$ \\
\hline \multicolumn{2}{|l|}{ CAR } & $1.20(0.55-2.27)$ \\
\hline \multicolumn{2}{|l|}{ NLR } & $1.99(1.50-2.40)$ \\
\hline \multicolumn{2}{|l|}{ PLR } & $119.08(95.16-147.18)$ \\
\hline \multirow{4}{*}{ Sacroiliitis, n (\%) } & Bilateral & $88(80.7 \%)$ \\
\hline & Right & $10(9.2 \%)$ \\
\hline & Left & $8(7.3 \%)$ \\
\hline & Absence & $3(2.8 \%)$ \\
\hline \multicolumn{3}{|c|}{$\begin{array}{l}\text { Ax-SpA: axial spondyloarthritis; BASDAl: Bath Ankylosing Spondylitis Disease } \\
\text { Activity Index; CRP: C-reactive protein; ESR: erythrocyte sedimentation rate; } \\
\text { CAR: CRP-to-albumin ratio; NLR: neutrophil-to-lymphocyte ratio; PLR: platelet- } \\
\text { to- Iymphocyte ratio; SD: standard deviation. }\end{array}$} \\
\hline
\end{tabular}

BASDAI score analysis revealed 61 (56 \%) patients with a score of $\geq 4$ (high activity) and 48 (44\%) patients with a score of $<4$ (low activity), respectively. There was a statistically significant differences in CRP, ESR, albumin, NLR and CAR values between the high- and low-activity patient groups ( $p<0.05$ ). The comparison of demographic and laboratory data of patients with Ax-SpA stratified based on disease activity is shown in Tablell.

Correlation analyses between BASDAl score and other clinical and laboratory parameters which indicate disease activity are presented in Table III. There was a statistically significant positive correlation between BASDAI and CAR, ESR, CRP and NLR. Further, it was noted a high correlation between CAR and CRP (rho: 0.998, p <0.001), low-to-moderate correlation between CAR and ESR (rho: 0.377, p <0.001), and CAR and NLR (rho: $0.347, p<0.001)$. 
Table II: Comparison of patients according to disease activity index (BASDAI).

\begin{tabular}{|l|l|l|l|}
\hline & $\begin{array}{l}\text { Low activity } \\
\text { BASDAl }<\mathbf{4}(\mathbf{n}=\mathbf{4 8})\end{array}$ & $\begin{array}{l}\text { High activity } \\
\text { BASDAI } \geq \mathbf{4}(\mathbf{n}=\mathbf{6 1})\end{array}$ & $\mathbf{p}$ \\
\hline Age, years (mean \pm SD) & $43 \pm 10.77$ & $46.62 \pm 10.32$ & 0.077 \\
\hline Male Gender, $\mathrm{n}(\%)$ & $25(52.1 \%)$ & $36(59.0 \%)$ & 0.469 \\
\hline Disease duration, years & $4(2-5)$ & $5(2.5-7)$ & 0.082 \\
\hline CRP, mg/L & $2.25(1.04-3.81)$ & $9.23(6.27-15.11)$ & $<0.001$ \\
\hline ESR, mm/h & $6(4-11.75)$ & $12(5-19)$ & 0.001 \\
\hline Albumin, g/dL & $4.58(4.46-4.71)$ & $4.36(4.18-4.58)$ & $<0.001$ \\
\hline CAR & $0.49(0.22-0.80)$ & $2.20(1.49-3.71)$ & $<0.001$ \\
\hline NLR & $1.86(1.42-2.14)$ & $2.12(1.75-2.53)$ & 0.015 \\
\hline PLR & $115.19(87.52-136.98)$ & $125.11(95.75-156.26)$ \\
\hline $\begin{array}{l}\text { BASDAl: Bath ankylosing spondylitis disease activity index; CRP: C-reactive protein; ESR: erythrocyte sedimentation rate; CAR: CRP-to-albumin ratio; NLR: } \\
\text { neutrophil-to-lymphocyte ratio; PLR: platelet-to- lymphocyte ratio; SD: standard deviation. }\end{array}$ \\
\hline
\end{tabular}

Table III: Correlation evaluations of BASDAI and laboratory parameters in Ax-SpA patients.

\begin{tabular}{|c|c|c|}
\hline & rho value & p-value \\
\hline CRP & $0.901^{* *}$ & $<0.001$ \\
\hline ESR & $0.326^{* *}$ & 0.001 \\
\hline CAR & $0.898^{* *}$ & $<0.001$ \\
\hline NLR & $0.327^{* *}$ & 0.001 \\
\hline PLR & 0.149 & 0.123 \\
\hline \multicolumn{3}{|c|}{$\begin{array}{l}\text { BASDAl: Bath ankylosing spondylitis disease activity index; CRP: C-reactive } \\
\text { protein; ESR: erythrocyte sedimentation rate; CAR: CRP-to-albumin ratio; } \\
\text { NLR: neutrophil-to-lymphocyte ratio; PLR: platelet-to- lymphocyte ratio. } \\
\text { Spearman korelasyon analizi }{ }^{* *} p<001 \text {. }\end{array}$} \\
\hline
\end{tabular}

Patients with normal CRP and ESR $(n=49)$ were selected and divided into two groups (BASDAI low activity $[<4]$; BASDAl high activity $[\geq 4]$ ); based on the evaluation of these groups, the median CAR of the low activity group ( $n=43$ ) was $0.41(0.20-0.73)$ and that of the high activity group was $0.84(0.55-0.95)(p=0.033)$. In addition there was a moderate correlation (rho: $0.410, p=0.003$ ) between BASDAI and CAR in this group.

\section{DISCUSSION}

Even in the era of new treatment strategies that make remission or "inactive disease" an achievable target in Ax-SpA, a persisting need for standardized and easily applicable and reliable tools for the evaluation of disease activity has been reported. ${ }^{16}$ In clinical studies, it is seen that the BASDAI composite score is commonly used to evaluate disease activity in Ax-SpA. CAR value has been investigated in many diseases as a value that reflects the systemic inflammation burden similar to NLR and PLR. ${ }^{9,10,17}$ In this study, which investigated the relationship between CAR value and BASDAI for the first time, there was a significant positive correlation between BASDAI with CAR, ESR, CRP, and NLR. A moderate correlation was also observed between BASDAI and CAR in the patient group with normal APR.

Similar to this study, a positive correlation was found between NLR and BASDAl in previous studies on patients with Ax-SpA. ${ }^{18}$ However, the authors could not find any study in the literature that evaluated the relationship between CAR and BASDAI. Previous studies have shown increased CAR values in cancer and intensive care patients; and have associated with disease inflammation. ${ }^{19,20}$ In addition, in a study by Bozkurt et al. on 35 healthy volunteers and 35 patients, who experienced uveitis episodes, it was reported that CAR could be an important parameter in determining the activation of uveitis. ${ }^{21}$ However, there are only a few studies on the role of CAR as an APR in autoimmune diseases. Yang et al. reported a correlation between CAR and DAS28 in patients with rheumatoid arthritis, and suggested that CAR may be used as an indicator to evaluate the disease activity in such cases. ${ }^{22}$ Similarly, Sunar et al. reported a positive but weak correlation among DAS28 - ESR and ESR with CAR. ${ }^{17}$ In their recent retrospective study on 32 patients with arthritis and 32 healthy controls, Takayasu found that CAR was significantly associated with disease activity, CRP, and ESR levels. ${ }^{12}$ In a study involving patients with antineutrophil cytoplasmic antibody-associated vasculitis, it was reported that at diagnosis, CAR was an independent predictor of all-cause mortality. ${ }^{23}$ Another study found a positive correlation between CAR and CRP and ESR in patients with rheumatoid arthritis. ${ }^{24} \mathrm{~A}$ study, which included patients with psoriasis reported that CAR could be used as an inflammatory biomarker of psoriatic arthritis in addition to being an inflammatory biomarker of psoriasis in patients with psoriasis treated with biological agent. ${ }^{25}$

BASDAI, a reliable and valid index in the evaluation of patients with Ax-SpA, is one of the most commonly used measures of disease activity. However, its application is subjective and may result in possible high activity values, as it is self-evaluated by the patients. It is also somewhat time consuming and hence less applicable in busy environments. It is believed that an objective tool is needed to evaluate disease activity. CAR appears to be advantageous as a quick, simple assessment method that positively correlates with BASDAI. In addition, being significant in detecting high disease activity in the group with normal APRs may be another advantage. Therefore, CAR may be recommended as a candidate for an additional disease activity index.

The first limitation of this study is that it was a single-centre study and the number of patients was not very large. Secondly, it was cross-sectional study and thirdly, other 
important indices used in the evaluation of disease activity in Ax-SpA (e.g., the ankylosing spondylitis disease activity score (ASDAS), ASDAS - CRP and ASDAS - ESR) were not included in the study, and correlation calculations could not be made. Finally, the effect of treatment on CAR and NLR values were not evaluated. Further detailed investigations should be conducted to determine the applicability of CAR as an early diagnostic marker of inflammation in Ax-SpA.

With the emerging new treatment modalities, the need for standard measurement methods that objectively indicate disease activity, can be applied easily, and are not time consuming is increasing. It is important that these methods are reliable methods that can be recognized by reimbursement institutions and include measurements that physicians can use practically in routine outpatient clinics.

\section{CONCLUSION}

CAR may be a new and practical measurement method in the follow-up of patients with Ax-SpA as it can be used quickly in routine outpatient conditions, does not contain a subjective parameter, does not increase the cost, and correlates with validated measurement method such as BASDAI. Therefore, CAR may be recommended as a candidate for an additional disease activity index.

\section{ETHICAL APPROVAL:}

The study protocol was approved by local ethics committee (Approval No. 102/3).

\section{AVAILABILITY OF DATA AND MATERIALS:}

The datasets used and/or analysed during the current study are available from the corresponding author on reasonable request.

\section{CONFLICT OF INTEREST:}

The authors declared no conflict of interest.

\section{AUTHORS' CONTRIBUTION:}

MP: Conception and design, data acquisition, interpretation, drafting, critical revision, final approval.

TID: Conception and design, data analysis, interpretation, drafting, critical revision, final approval.

\section{REFERENCES}

1. Maksymowych WP. Biomarkers for diagnosis of axial spondyloarthritis, disease activity, prognosis, and prediction of response to therapy. Front Immunol 2019; 10:305. doi:10.3389/fimmu.2019.00305.

2. Poddubnyy D, Haibel H, Listing J. Baseline radiographic damage, elevated acute-phase reactant levels, and cigarette smoking status predict spinal radiographic progression in early axial spondylarthritis. Arthritis Rheum 2012; 64(5):1388-98. doi:10.1002/art.33465.

3. Çetin P, Solmaz D, Sari İ. Aksiyal Spondiloartrit. Türkiye Klinikleri Romatoloji-Özel Konular 2015; 8:24-31.

4. Colglazier $\mathrm{CL}$, Sutej PG. Laboratory testing in the rheumatic diseases: A practical review. South Med J 2005; 98(2):185-91. doi:10.1097/01.Smj.0000153572.22346.E9.

5. Castell JV, Gómez-Lechón MJ, David M. Acute-phase response of human hepatocytes: Regulation of acute-phase protein synthesis by interleukin-6. Hepatology 1990; 12(5):1179-86. doi:10.1002/hep.1840120517.

6. Domínguez de Villota E, Mosquera JM, Rubio JJ. Association of a low serum albumin with infection and increased mortality in critically ill patients. Intensive Care Med 1980; 7(1): 19-22. doi:10.1007/bf01692917.

7. Garrett S, Jenkinson T, Kennedy LG. A new approach to defining disease status in ankylosing spondylitis: The bath ankylosing spondylitis disease activity index. J Rheumatol 1994; 21(12):2286-2291.

8. Kılıç $S$, Işık S, Hiz MM. The ischemia modified albumin and mean platelet volume levels in patients with Behçet's disease. Postepy Dermatol Alergol 2016; 33(5):345-8. doi:10.5114/ada.2016.62840.

9. Erre GL, Paliogiannis P, Castagna F. Meta-analysis of neutrophil-to-lymphocyte and platelet-to-lymphocyte ratio in rheumatoid arthritis. Eur J Clin Invest 2019; 49(1): e13037. doi:10.1111/eci.13037.

10. Kalyoncuoglu M, Durmus G. Relationship between C-reactive protein-to-albumin ratio and the extent of coronary artery disease in patients with non-ST-elevated myocardial infarction. Coron Artery Dis 2020; 31(2):130-6. doi:10. 1097/mca.0000000000000768.

11. Kocatürk M, Kocatürk Ö. Assessment of relationship between C-reactive protein to albumin ratio and 90-day mortality in patients with acute ischaemic stroke. Neurol Neurochir Pol 2019; 53(3):205-211. doi:10.5603/ PJNNS. a2019.0020.

12. Seringec Akkececi N, Yildirim Cetin G, Gogebakan H. The Creactive protein/albumin ratio and complete blood count parameters as indicators of disease activity in patients with takayasu arteritis. Med Sci Monit 2019; 25:1401-9. doi:10.12659/msm.912495.

13. Rudwaleit M, Van Der Heijde D, Landewé R. The development of assessment of spondyloarthritis international society classification criteria for axial spondyloarthritis (part II): Validation and final selection. Ann Rheum Dis 2009; 68(6):777-83 doi: 10.1136/ ard.2009.108233.

14. Akkoc Y, Karatepe AG, Akar S. A Turkish version of the bath ankylosing spondylitis disease activity index: Reliability and validity. Rheumatol Int 2005; 25(4):280-4. doi:10.1007/ s00296-003-0432-y.

15. Murat Hayran MH. Sağlık araştırmaları için temel istatistik. Omega Araştırma 2011;

16. Proft F, Muche B, Spiller L. Performance of the ankylosing spondylitis disease activity score based on a quick quantitative C-reactive protein assay in patients with axial spondyloarthritis. Joint Bone Spine 2020; 87(1):69-73. doi:10. 1016/j.jbspin.2019.07.007.

17. Sunar I, Ataman Ş. Serum C-reactive protein/albumin ratio in rheumatoid arthritis and its relationship with disease activity, physical function, and quality of life. Arch Rheumatol 2020; 35(2):247-53. doi:10.46497/Arch Rheumatol.2020.7456. 
18. Xu S, Ma Y, Wu M. Neutrophil lymphocyte ratio in patients with ankylosing spondylitis: A systematic review and meta-analysis. Mod Rheumatol 2020; 30(1):141-8. doi:10.1080/ 14397595.2018.1564165.

19. Deng TB, Zhang J, Zhou YZ. The prognostic value of C-reactive protein to albumin ratio in patients with lung cancer. Medicine (Baltimore) 2018; 97(50):e13505. doi:10.1097/ md.0000000000013505.

20. Park JE, Chung KS, Song JH. The C-reactive protein/albumin ratio as a predictor of mortality in critically III patients. J Clin Med 2018; 7(10). doi:10.3390/jcm7100333.

21. Bozkurt E, Muhafiz E, Sengul D. Can the CRP/albumin ratio be used as a new indicator of activation in patients with uveitis? Ocular Immunol Inflamm 2020; 1-6. doi:10.1080/ 09273948.2020.1714061.

22. Yang WM, Zhang WH, Ying $\mathrm{HQ}, \mathrm{Xu}$ YM, Zhang J, Min $\mathrm{QH}$, et al. Two new inflammatory markers associated with disease activity score-28 in patients with rheumatoid arthritis:
Albumin to fibrinogen ratio and C-reactive protein to albumin ratio. Int Immunopharmacol 2018; 62:293-8. doi:10.1016/j.intimp.2018.07.007.

23. Moon JS, Ahn SS, Park YB, Lee SK, Lee SW. C-reactive protein to serum albumin ratio is an independent predictor of all-cause mortality in patients with ANCA-associated vasculitis. Yonsei Med J 2018; 59(7):865-71. doi:10.3349/ ymj.2018.59.7.865.

24. He Y, Tang J, Wu B. Correlation between albumin to fibrinogen ratio, C-reactive protein to albumin ratio and Th17 cells in patients with rheumatoid arthritis. Clinica Chimica Acta 2020; 500:149-54. doi: 10.1016/j.cca. 2019.10.009.

25. Tamer F, Avcl E. Serum C-reactive protein to albumin ratio as a novel inflammation biomarker in psoriasis patients treated with adalimumab, ustekinumab, infliximab, and secukinumab: A retrospective study. Croat Med J 2020; 61(4):333-7. doi:10.3325/cmj.2020.61.333. 